\title{
Phenomenological modeling of anisotropy induced by evolution of the dislocation structure on the macroscopic and microscopic scale
}

\author{
Till Clausmeyer • Ton van den Boogaard • \\ Muhammad Noman • Grygoriy Gershteyn • \\ Mirko Schaper · Bob Svendsen · Swantje Bargmann
}

Received: 9 June 2010 / Accepted: 4 December 2010 / Published online: 6 January 2011

(C) The Author(s) 2011. This article is published with open access at Springerlink.com

\begin{abstract}
This work focuses on the modeling of the evolution of anisotropy induced by the development of the dislocation microstructure. A model formulated at the engineering scale in the context of classical metal plasticity and a model formulated in the context of crystal plasticity are presented. Images obtained by transmission-electron microscopy (TEM) show the influence of the strain path on the evolution of anisotropy for the case of two common materials used in sheet metal forming, DC06 and AA6016-T4. Both models are capable of accounting for the transient behavior observed after changes in loading path for fcc
\end{abstract}

T. Clausmeyer $(\varangle) \cdot$ M. Noman $\cdot$ S. Bargmann Institute of Mechanics, TU Dortmund University, Leonhard-Euler-Straße 5, 44225, Dortmund, Germany e-mail: till.clausmeyer@udo.edu

S. Bargmann

e-mail: swantje.bargmann@udo.edu

T. van den Boogaard

Faculty of Engineering Technology, University of Twente,

217, 7500 AE, Enschede, The Netherlands

e-mail: A.H.vandenBoogaard@ctw.utwente.nl

G. Gershteyn · M. Scharper

Institute of Material Science, Leibniz Universität Hannover,

An der Universität 2, 30823, Garbsen, Germany

G. Gershteyn

e-mail: gersteyn@iw.uni-hannover.de

M. Scharper

e-mail: schaper@iw.uni-hannover.de

B.Svendsen

Chair of Material Mechanics, RWTH Aachen University,

Schinkelstraße 2, 52062, Aachen, Germany

e-mail: bob.svendsen@rwth-aachen.de and bcc metals. The evolution of the internal variables of the models is correlated with the evolution of the dislocation structure observed by TEM investigations.

Keywords Material modeling •

Microstructural evolution • Cross hardening •

Induced flow anisotropy

\section{Introduction}

In general, metal forming processes involve large strains and severe strain-path changes. Large plastic strains lead in many metals to the development of persistent dislocation structures resulting in strong flow anisotropy. This induced anisotropic behavior manifests itself in the case of a strain-path change through different stress-strain responses depending on the type of strain-path change. Since metal forming processes involve both large plastic strains and severe strainpath changes, an adequate modeling of this induced flow anisotropy is crucial for the proper prediction of residual stresses and, consequently, of the amount of springback in structural components. Starting with Ghosh and Backofen [8] who investigated the influence of different strain paths on the deformation behavior and observed a variation of the work-hardening rate in sheet metals depending on the pre-deformation, Hasegawa et al. [10] related the decrease in the rate of work-hardening after a reverse in strain direction to the changes in the substructure, namely the partial dissolution of cells and a slight decrease in the corresponding dislocation density. Strauven and Aernoudt [32] also followed the changes in the microstructure and related it to the transient regions of work-hardening observed 
in tension-compression tests. $[28,29,35]$ extended the investigation of the microstructural changes to the case of strain path changes from tension to shear and related it to the characteristic changes in yield stress and workhardening behavior. Estrin et al. [6] proposed a model on the dislocation-structure level, in order to reproduce the experimentally found stress-strain curves based on an earlier approach by Mughrabi [19]. In particular, these models account for the changes in dislocation structures, resulting in early re-yielding after load reversal accompanied by a period of work-hardening stagnation and cross-hardening after orthogonal strainpath changes. In this context, studies focusing either on the characterization of the changes in the dislocation microstructure and texture evolution (e.g. by $[24,25]$ ) or on the development of a phenomenological model for the macroscopic stress-strain behavior by $[13,33]$ have been carried out. These allow to interpret the macroscopic material behavior of polycrystalline metals under strain-path changes at large deformation. Among the material models accounting for these additional effects, that of Teodosiu and $\mathrm{Hu}[33,34]$ has been used by a number of authors e.g. $[3-5,11]$ to model the induced anisotropic hardening behavior and investigate its effect on forming processes $[4,15,36]$.

In what follows, we refer to this model for simplicity as the Teodosiu model. In this model, evolving structure tensors are used to account for directional hardening effects resulting from the development of persistent dislocation structures during monotonic loading and from their reorganization upon changes in loading direction. A recent model presented in [26] formulated on the engineering scale also accounts for transient behavior observed in cross-tests by modifying the shape of the yield surface in dependence of the strain path. Similar to the Teodosiu model the evolution of the dislocation structure is taken into account by tensor-valued internal variables. In this contribution, this model is referred to as the yield surface model for directional hardening (YSDH).

Models formulated in the context of crystal plasticity enable a less abstract interpretation of the internal variables and the correlation with the observed changes in the dislocation structures. In [27] a crystal-plasticity based model which introduces three different dislocation densities on the glide system level was applied to model the deformation behavior of interstitial free steel subjected to shear followed by reversed shear and tension-followed by shear loading. Based upon this model Holmedal et al. [12] developed a model which introduces a phenomenological hardening law on the glide system level in order to account for the deformation behavior of a 3000 series aluminum alloy. In what
Table 1 Chemical composition of DC06 according to [21]

\begin{tabular}{llllllll}
\hline $\mathrm{C}$ & $\mathrm{Si}$ & $\mathrm{Mn}$ & $\mathrm{P}$ & $\mathrm{S}$ & $\mathrm{Al}$ & $\mathrm{N}$ & $\mathrm{Ti}$ \\
\hline 0.003 & 0.018 & 0.137 & 0.013 & 0.010 & 0.035 & 0.0027 & 0.079 \\
\hline
\end{tabular}

All values are given as weight percentage and were determined by ThyssenKrupp Steel Europe by means of an analysis of one single specimen

follows, this model and in particular the glide system hardening laws are referred to as the Holmedal model.

In the current work the deformation behavior of the engineering materials, AA6016-T4 and DC06 is investigated using a biaxial tester. The work focuses on modeling of the transient behavior observed in cross-tests. In particular, plane strain tension to simple shear loading is considered and specimen subjected to this sequence are analyzed using electron microscopy. The macroscopic metal plasticity model and microscale crystal plasticity model using the Holmedal hardening formulation are presented and the results of parameter identification for AA6016-T4 and DC06 are shown for the YSDH model. Finally, the correlation between both models and the observed evolution of the dislocation structure is investigated by analyzing the evolution of internal variables.

\section{Materials tested: DC06 and AA6016-T4}

Table 1 gives the chemical composition of the interstitial free steel DC06 delivered by ThyssenKrupp Steel Europe AG according to [21]. After cold-rolling the material was annealed and subjected to a final skin-pass by the manufacturer. The average Young's Modulus $E$ over the three directions $0^{\circ}, 45^{\circ}$ and $90^{\circ}$ with respect to the rolling direction (RD) is determined as 181,000 $\mathrm{MPa}$ with Poisson's ratio $v=0.3$ according to [23]. The initial texture is a fiber texture with the $<111>$ direction oriented parallel to the sheet normal direction. The average grain size is $20 \mu \mathrm{m}$ with single grain sizes ranging from 5 to $60 \mu \mathrm{m}$. The sheet thickness is 1.00 $\mathrm{mm}$. Table 2 shows the chemical composition of the aluminum alloy (thickness $1.00 \mathrm{~mm}$ ) delivered by Novelis according to [22]. The microstructure of the alloy in the as-received state is well recrystallized. A low density of precipitates is observed within the grains. The average Young's Modulus $E$ over the three directions $0^{\circ}, 45^{\circ}$ and $90^{\circ}$ with respect to rolling direction is determined

Table 2 Chemical composition of AA6016-T4 according to [22]

\begin{tabular}{lllllll}
\hline $\mathrm{Si}$ & $\mathrm{Fe}$ & $\mathrm{Cu}$ & $\mathrm{Mn}$ & $\mathrm{Mg}$ & $\mathrm{Cr}$ & $\mathrm{Zn}$ \\
\hline $1.0-1.5$ & 0.5 & 0.2 & 0.2 & $0.25-0.6$ & 0.1 & 0.2 \\
\hline
\end{tabular}


as $68000 \mathrm{MPa}$ with Poisson's ratio $v=0.33$ according to [23]. A fiber texture with the $<111>$ direction oriented parallel to the rolling direction can be observed in the undeformed material. The grains tend to be elongated with characteristic lengths between 100-300 $\mu \mathrm{m}$ and widths of $20-70 \mu \mathrm{m}$. All specimen, aluminum and steel, used in this work were manufactured from the same batch of material.

\section{Material testing}

Test setup

For the experimental work a biaxial testing equipment developed at the Faculty of Engineering Technology at the University of Twente, Netherlands was used to subject specimen shown in Fig. 1 to planar states of deformation. Even though plane strain tension and sequences of forward to reverse shear were carried out in order to characterize the two materials in detail, in the current work the focus lies on cross-tests, i.e. sequences from plane strain tension to simple shear. In addition uniaxial tension tests were performed in order to determine the r-values. A vertical and a horizontal actuator can be used to deform the specimen. If only the vertical actuator is activated, the specimen is deformed in plane strain tension. If only the horizontal actuator is used, the specimen is deformed in simple shear as can be seen in Fig. 1. Mixed plane strain tension/simple shear loading is possible if both actuators are active simultaneously. In addition, arbitrary sequences of attainable states of deformation are also possible.

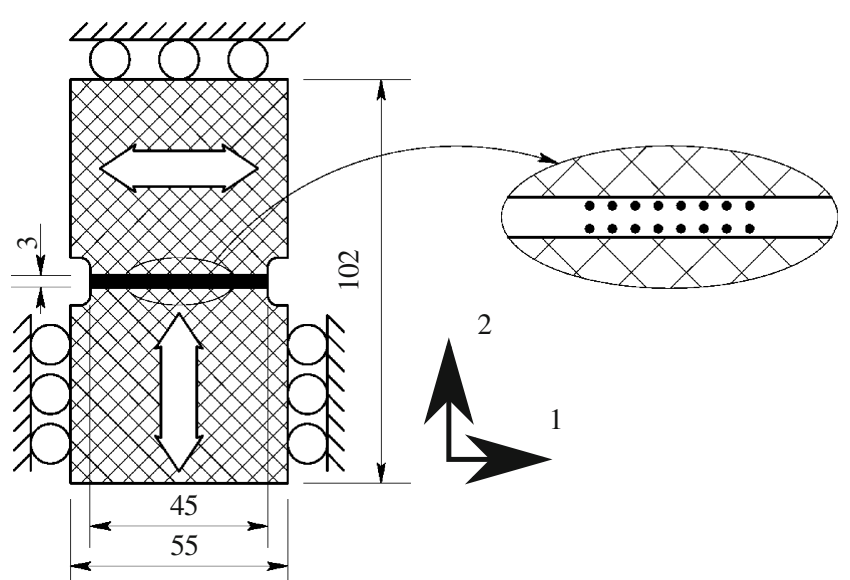

Fig. 1 Biaxial test setup. Geometry of the tension-shear specimen and the measurement region of height $3.0 \mathrm{~mm}$ and width $45.0 \mathrm{~mm}$. The checkered region indicates the actual specimen and the black area marks the actual deformation zone. The tension direction is direction 2 and the shear direction is direction 1
The deformation field is obtained by optical measurement of an array of painted dots in the center of the deformation zone of the specimen. Since the deformation field in the deformation zone is homogeneous for the level of straining investigated in this work, the relative motion of the dots determines the deformation field. Components of the deformation gradient $F_{i j}=$ $\partial x_{i} / \partial X_{j}$ are used to characterize the deformation of the specimen. $x_{i}$ denotes the current position of a material point and $X_{j}$ denotes the reference position of a material point. The horizontal and vertical force are recorded such that, together with the known geometry, the Cauchy stresses $T_{22}$ and $T_{12}$ can be computed. Further details concerning the experimental setup can be found in $[26,37,38]$. In all experiments the strain rate was set to $10^{-3} 1 / \mathrm{s}$.

\section{Test results}

The stress vs. strain curves obtained in plane strain to simple shear tests obtained on the biaxial tester are shown in Figs. 2 and 3 for DC06 and AA6016T4, respectively. Average r-values are computed by evaluation of the ratios of total plastic strain for the beginning of plastic deformation (i.e., $\epsilon_{p, 22}=0.002$ ) to the uniform strain, the strain that occurs until necking starts. Here, $\epsilon_{p, 22}$ refers to the plastic part of the normal strain.

\section{DC06}

The average $r$-values in rolling direction, in $45^{\circ}$ with respect to the rolling direction and transverse direction

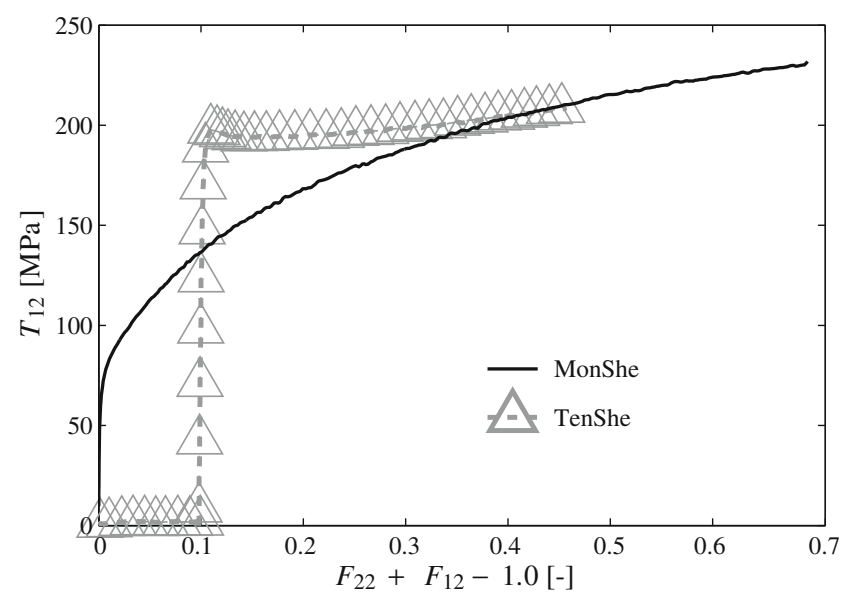

Fig. 2 Cauchy shear stress $T_{12}$ over $F_{22}+F_{12}-1$ for a plane strain tension to shear experiment (TenShe) and monotonic simple shear (MonShe) for DC06. The amount of pre-strain was $10.0 \%$ in rolling direction 


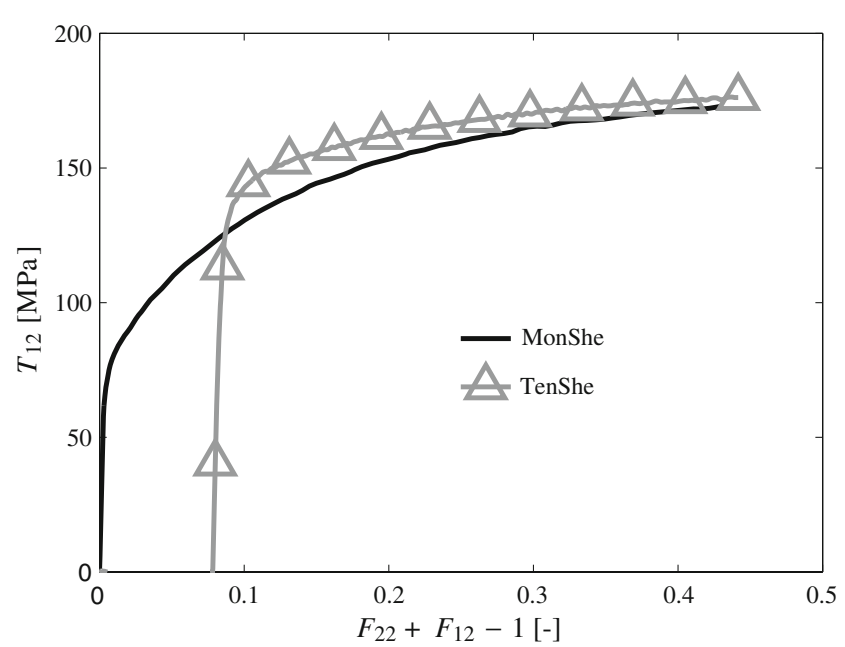

Fig. 3 Cauchy shear stress $T_{12}$ over $F_{22}+F_{12}-1$ for a plane strain tension to shear experiment (TenShe) and monotonic simple shear (MonShe) for AA6016-T4. The amount of pre-strain was $7.8 \%$ in rolling direction

for DC06 are determined as $r_{0}=2.31, r_{45}=1.95$ and $r_{90}=2.77$, respectively. Forward to reverse shear tests show that the material exhibits a clear Bauschinger effect, characterized by early re-yielding after load reversal. In addition the material exhibits distinct crosshardening during the loading path change from plane strain tension in rolling direction to simple shear, characterized by the distinct overshoot in shear stress (here: approximately $65 \mathrm{MPa}$ ) compared to the monotonic simple shear case. The magnitude of the overshoot increases with increasing pre-strain. The amount of prestrain for the experiment shown in Fig. 2 is given by $F_{22}=1.10$.

\section{AA6016-T4}

The average r-values for AA6016-T4 in rolling direction, in $45^{\circ}$ with respect to the rolling direction and transverse direction are determined as $r_{0}=0.630, r_{45}=$ 0.409 and $r_{90}=0.771$, respectively. Forward to reverse shear tests show that the material exhibits a moderate Bauschinger effect. The cross-hardening is less distinct for AA6016-T4 than for DC06. For a pre-strain of $F_{22}=1.078$ (Fig. 3), the overshoot in shear is about 15 MPa or less than $10 \%$.

\section{Microstructural investigation}

Undeformed and deformed specimen obtained in the mechanical tests are investigated using transmissionelectron microscopy. In order to obtain TEM-foils flat disks with a diameter of $3 \mathrm{~mm}$ and a thickness of approximately $500 \mu \mathrm{m}$ were cut from the center of the deformation zone of the deformed sample (see Fig. 1) by wire eroding. These disks were mechanically thinned and polished from both sides with emery paper of grades 320-4,000 until a thickness of $100 \mu \mathrm{m}$ and roughness $R_{a}<1 \mu \mathrm{m}$ according to [20] was obtained. During this and subsequent processing care is taken that only small pressure is exerted such that additional deformation to the preparation process is prevented.

Special marks parallel to the rolling, shearing and tensile direction were printed on the foils in order to reproduce this direction on the TEM images. The TEMfoils were electropolished with an electrolyte consisting of $120 \mathrm{ml} 40 \%$ perchloric acid, $440 \mathrm{ml}$ butoxyethanol and $440 \mathrm{ml} \mathrm{100 \%} \mathrm{acetic} \mathrm{acid} \mathrm{at} \mathrm{a} \mathrm{current} \mathrm{density} \mathrm{of} 100$ $\frac{\mathrm{mA}}{\mathrm{cm}^{2}}$ using platinum electrodes in a Stuers Tenu Pol 5 to the point of perforations. By-products (i.e. oxide film, single droplets of the rinsing agent) were removed from the electropolished surface by subjecting the foils to a 10-15 min ion milling treatment on a Gatan Duo-Mill 600 with a voltage of $5 \mathrm{kV}$ under an angle of $10-15^{\circ}$. Microstructural investigations are performed on the soobtained foils on a JEOL JEM2010 electron microscope with a $200 \mathrm{kV}$ electron gun. For the preparation of the aluminum samples, the preparation procedure of the TEM sample is slightly modified. A different electrolyte consisting of $30 \%$ nitric acid and ethanol is used. After the electropolishing, the sample is dried with nitrogen gas at $-25^{\circ} \mathrm{C}$ and the further processing is performed as for the steel case.

\section{TEM-images after tension \& analysis}

In the description of the dislocation microstructure, the following terms will be used for structural elements: (i) cells are defined as regions of low dislocation density surrounded by lines of higher dislocation density, within the cells the dislocation is homogeneously distributed, usually neighboring cells have an misorientation smaller than $0.2-0.8^{\circ}$; (ii) cell walls are regions of higher dislocation density surrounding the cells, the misorientation from cell interior to the cell wall can be as large as $3^{\circ}$; (iii) cell-block boundaries are dense dislocation walls which surround an area of dislocation cells, they have shapes like parallelepipeda and confine planar persistent dislocation structures.

\section{DC06}

In addition to specimen subjected to plane strain tension, specimen manufactured from DC06 subjected to uniaxial tension were investigated. There are certain 
characteristic features of the evolution of the dislocation microstructure common to uniaxial and plane strain tension, alike. During tension, DC06 tends to form cell-block boundaries initially predominantly associated with $\{110\}$ glide systems. As deformation continues cell-block boundaries belonging to $\{112\}$ systems can be observed. Starting from $15 \%$ tension, cell-block boundaries oriented on $\{123\}$ planes also appear. Formation of cell-block boundaries is observed starting with $5 \%$ tensile strain. However, at strains between 5 and $8 \%$, cells and planar persistent structures coexist. Also at strains between 5 and $12 \%$ tension, dislocations tangles can be observed. It seems that these appear in certain parts of the sample while deformation is increased in the mentioned range, while simultaneously other tangles seem to disappear. With increasing tensile deformation the misorientation angle between cell interior and cell-block boundaries increases, finally resulting in sharp boundaries referred to as blade-like plates by [30] on a larger scale. Figure 4 shows a region dominated by cell-block boundaries for uniaxial tension. The cell-block boundaries are usually roughly aligned with the macroscopic tensile axis (TenD). In plane strain tension cell block-boundaries exhibit higher curvature and a second family of cell-block-boundaries occurs more often compared to uniaxial tension. The details of this comparison will be published in a future work focusing on metallography.

\section{AA6016-T4}

After a deformation of $10 \%$ plane strain the electronmicroscopical investigation of the aluminum alloy

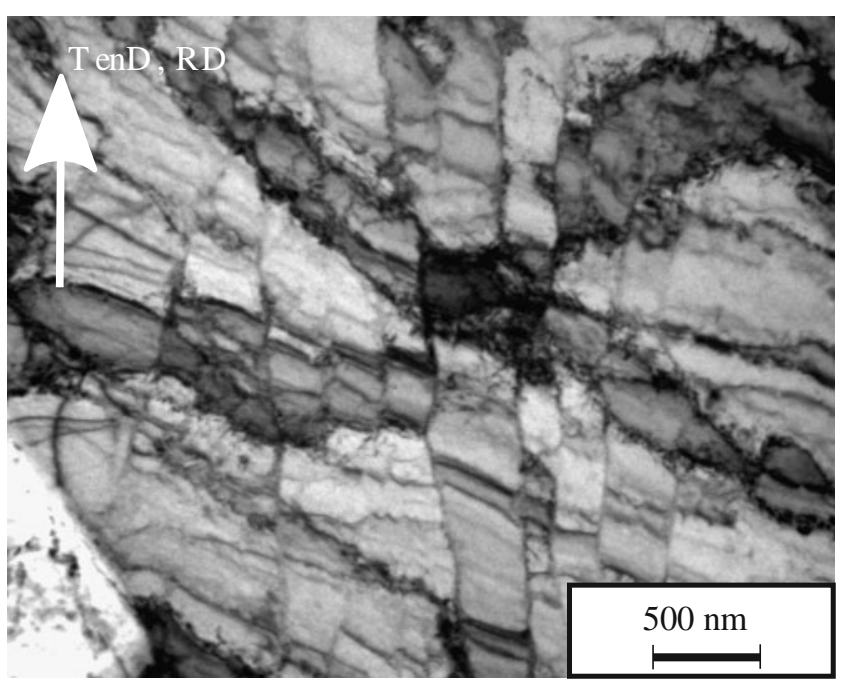

Fig. 4 Fragmentation after 5\% tensile strain in rolling direction in uniaxial tension for DC06. TenD denotes the tensile direction

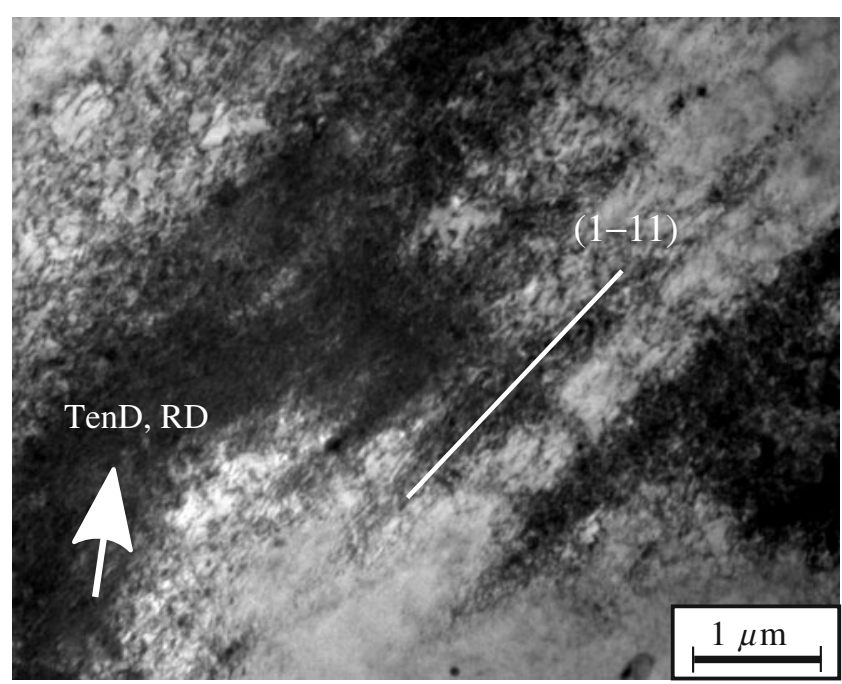

Fig. 5 Dislocation structures after plane strain tension in AA6016 for tension in rolling direction up to $10 \%$

showed that regions where dislocation walls and dislocations networks form can be observed in the microstructure. The dislocation walls are inclined $\pm 40^{\circ}$ to the macroscopic tension direction. In addition grains exhibiting a cell structure can be found. With increasing deformation up to about $20 \%$ tension, the dislocation density increases. Most dislocation walls are still inclined $\pm 40^{\circ}$ to the macroscopic tension axis. The formation of dislocation structures is intensified. Often two families of dislocation walls can be found in the microstructure. Note that these walls are not parallel to the tensile direction, but to traces of $\{111\}$ slip planes, see e.g. Fig. 5.

\section{TEM-images after shear $\&$ analysis}

\section{DC06}

For the case of monotonic shear deformation of DC06, the results of earlier investigations by [7, 28, 35] and for interstitial free steels can be confirmed. Two families of cell-block boundaries are predominant, either parallel or orthogonal to the shearing direction. For large amounts of deformation a misorientation of up to $1^{\circ}$ can be observed within the cell-block-boundaries having a maximum distance of $0.5 \mu$ (Fig. 6).

\section{AA6016-T4}

The following characteristic features can be observed for simple shear deformation in AA6016-T4: after a plastic deformation of $10 \%$ shear the cells possess an approximate size of $1 \mu \mathrm{m}$. Figure 7 shows that the 


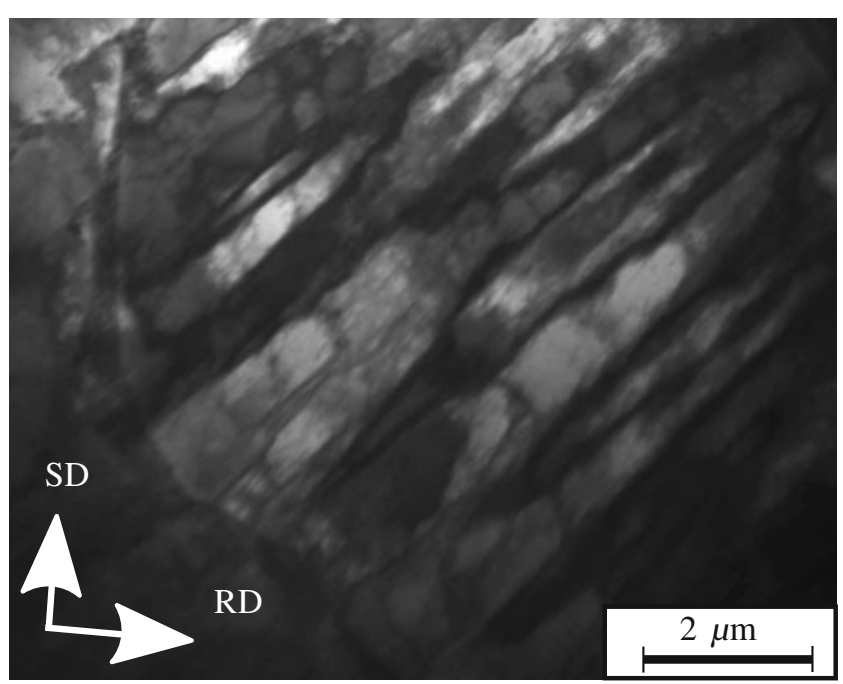

Fig. 6 Dislocation structures after 30\% simple shear in DC06. $\mathrm{SD}$ is orthogonal to the rolling direction

size of single cells after $30 \%$ shear is in the range of $0.25-2 \mu \mathrm{m}$. In addition to the formation of dislocation walls and dislocation networks, single dislocations can be observed. As in the case of tension, dislocation nets in the longitudinal planes are inclined about $\pm 40^{\circ}$ to the rolling or tension direction in case these align.

With increasing shear deformation up to a shear strain of $20 \%$ the density of dislocations increases. In addition, the formation of dislocation walls and dislocation networks intensifies. Two families of networks can be identified. One family of networks is formed with an angle of inclination of $35^{\circ}$ with respect to the

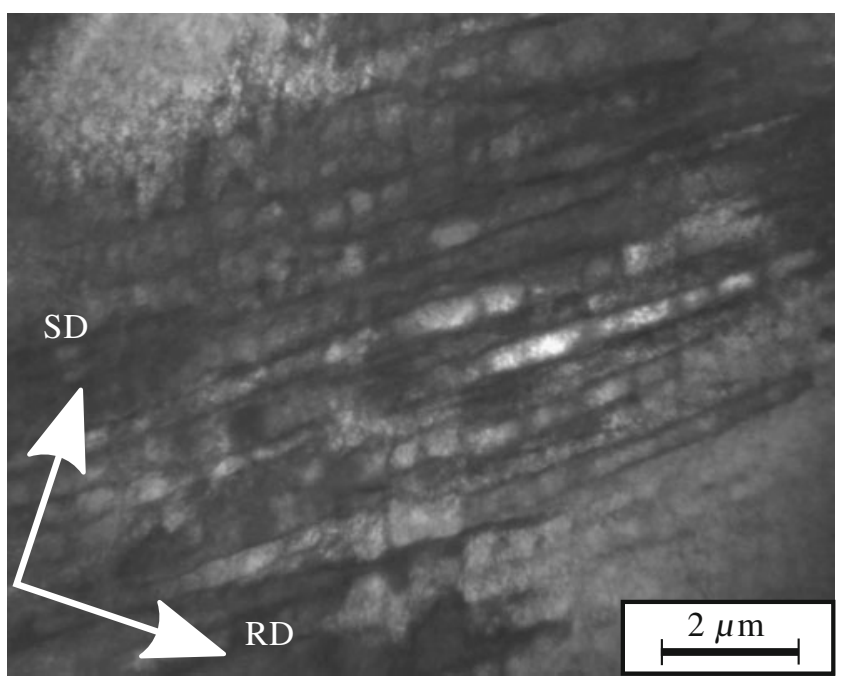

Fig. 7 Dislocation structures after simple shear in AA6016 orthogonal to rolling direction up to $30 \%$ macroscopic shear direction. However, in some grains microbands begin to form as can be seen in Fig. 7 .

TEM-images after tension-shear \& analysis

\section{DC06}

For DC06 for the case of a plane strain tension to simple shear sequence, in grains with a Schmidt factor between 0.25 and 0.5 the dislocation microstructure can be described as a grid-like structure formed by the cellblock boundaries which were formed during the plane strain tension deformation and are roughly aligned with the tensile direction as can be seen from Fig. 8 for the central grain. EBSD measurements revealed that over $50 \%$ of all grains have a Schmidt factor in this range. It can be assumed that the cell-block boundaries parallel to the shear direction in Fig. 8 are former microbands which have cut through the planar dislocation structure from the tensile loading and have evolved into cellblock boundaries with increasing shear load.

\section{AA6016-T4}

The region of the AA6016-T4 sample deformed in the tension-to-shear sequence in Fig. 9 shows less pronounced planar dislocation structures compared to the DC06 case. But careful inspection reveals that there is also a faint network of dislocation structures in two distinctly different directions, here indicated by the dashed white lines. It can be assumed that the traces

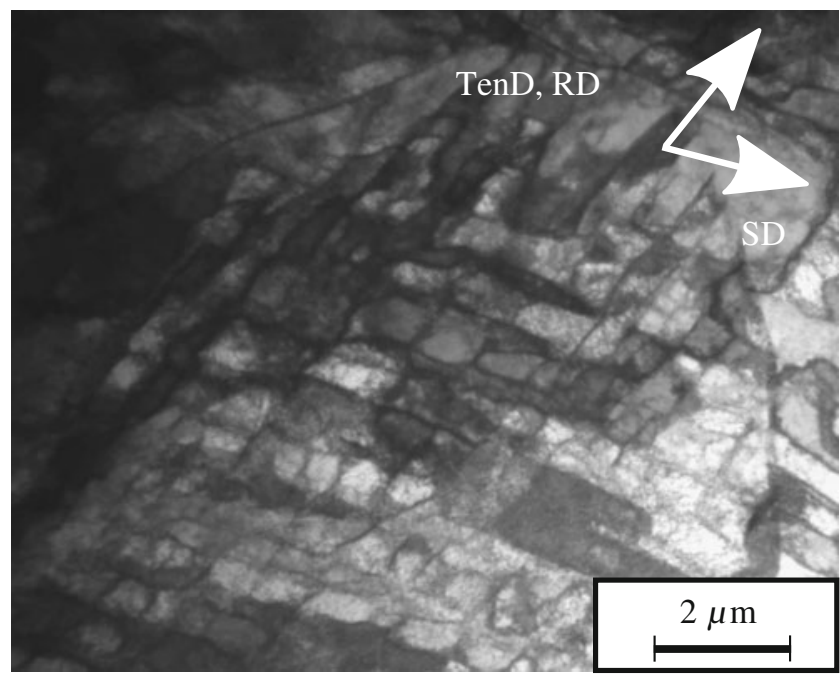

Fig. 8 Microstructure after a sequence of plane strain tension $\left(F_{22}=1.11\right)$ in rolling direction and simple shear $\left(F_{12}=0.35\right)$ for DC06 


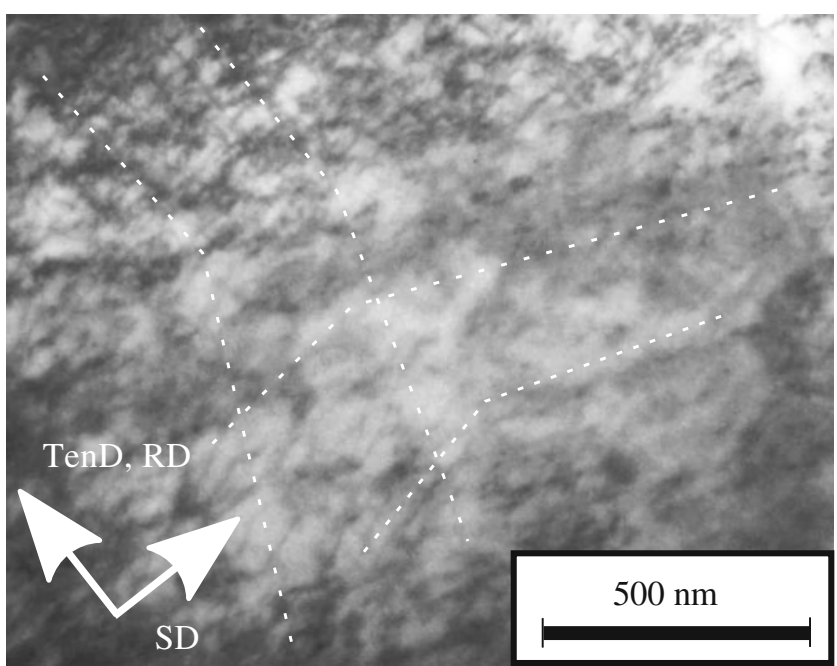

Fig. 9 Microstructure after a sequence of plane strain tension $\left(F_{22}=1.08\right)$ in rolling direction and simple shear $\left(F_{12}=0.18\right)$ for AA6016-T4

of walls running from the upper left corner to the bottom of the image are associated with the fist stage of deformation. Consequently, the traces of walls running from the lower left corner to the right side are due to the shear deformation.

\section{Correlation microstructure-stress-strain transients}

The evolution of distinct elements of the microstructure for the DC06 material can be linked to the transients observed in the stress-strain relations for the mechanical tests presented in the mechanical test section. Figure 10 shows schematically how the transient regions in stress strain space are linked to characteristic sections of the dislocation microstructure. Of particular interest is the increase in the yield stress upon the change from plane strain tension to simple shear which can be understood as the macroscopic counterpart of the activation of new glide systems which had been latent during the tension phase as described by e.g. [28]. Microbands which form parallel to the most active glide systems interact with the planar persistent dislocation structure formed during tension. The work-softening followed by this can be understood as structural weakening of the planar persistent dislocations structures formed during prestraining [35]. In DC06 the planar persistent dislocation structures seem to be more pronounced than in AA6016-T4 for comparable states of deformation. The higher intensity of these dislocation structures in DC06 corresponds to the stronger cross-hardening effect in DC06 compared to AA6016-T4. Here, intensity refers to the thickness of dense dislocation walls and the spac-

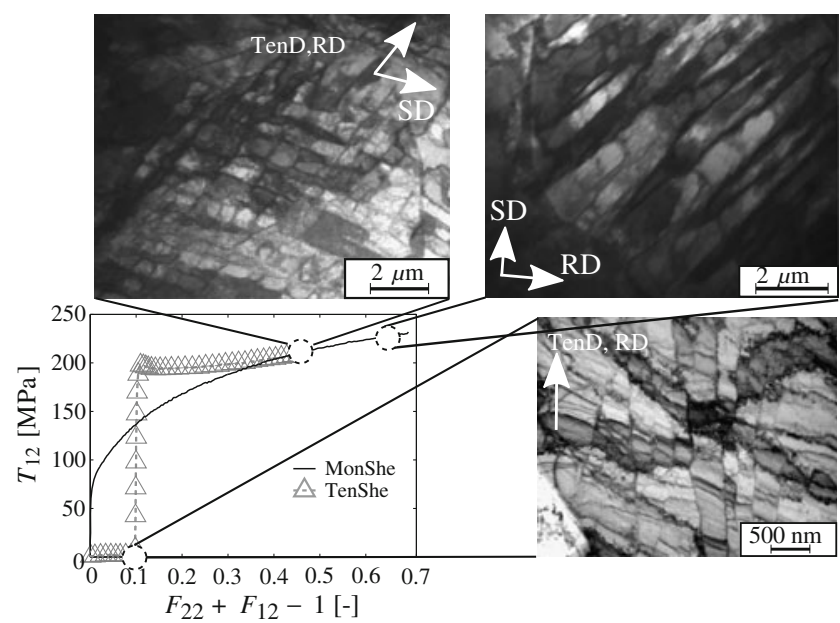

Fig. 10 Illustration for the correlation of the evolution of the dislocation microstructure and macroscopic stress-strain behavior in DC06. The abscissa of the graph shows $F_{22}+F_{12}-1.0$ for a monotonic shear test (MonShe) and a plane strain tension to shear test (TenShe)

ing of single walls. It is assumed that cross-hardening occurs if these strucures are present in the material and a loading path change occurs. Thus, it is expected and confirmed by the experiments that the cross-hardening effect in DC06 is higher in DC06 than in AA6016-T4 as Figs. 2 and 3 show. Also, fine dispersions of precipitates in the aluminum alloy seem to retard the tendency to form dense-dislocation walls.

\section{Model formulation}

Both models to be presented in the following are formulated in order to account for the transients in stressstrain relation after strain path changes induced by the evolution of the dislocation structure as shown in the previous sections. First, the engineering scale model will be presented and its characteristic features discussed. Next, the microscale model and the similarities and differences between the two modeling approaches will be presented.

\section{Macroscale metal plasticity model}

In the YSDH model, internal variables are used to model the effect of pile-ups of dislocations, the interaction of long-range stress fields introduced by dislocations and the formation of persistent dislocation 
structures. In the context of the phenomenological representation of evolving hardening behavior at this scale in terms of changes in the size, center and shape of the yield surface offers the means to characterize the behavior of the steels of interest during complex, nonproportional loading processes present in many technological processes (e.g., deep-drawing). The model is aimed at being used in the FE-simulation of such processes, mainly for bcc material. The challenge in modeling lies in the connection of such changes in the yield surface geometry with the underlying microscopic and physical mechanisms of grain and dislocation microstructural development in polycrystalline metals, which have been presented in the previous section. One basic expectation in this regard is that the grain microstructure in sheet metals is determined almost solely by the rolling process. Forming processes like cup drawing are expected to result in little or no change in this microstructure, at least for the case of bcc metals. Consequently, during forming processes, evolution of anisotropy is generally expected to be due almost solely to an evolving dislocation microstructure at the grainor grain-cluster level. This is the focus of this model.

Both models to follow are formulated in incremental form within the framework of the inelastic multiplicative decomposition $\boldsymbol{F}=\boldsymbol{F}_{\mathrm{E}} \boldsymbol{F}_{\mathrm{P}}$ of the deformation gradient $\boldsymbol{F}$. For metals, the assumption of small elastic strain implies $\boldsymbol{U}_{\mathrm{E}} \approx \boldsymbol{I}$, and thus $\boldsymbol{F}_{\mathrm{E}} \approx \boldsymbol{R}_{\mathrm{E}}$ in the context of the polar decomposition $\boldsymbol{F}_{\mathrm{E}}=\boldsymbol{R}_{\mathrm{E}} \boldsymbol{U}_{\mathrm{E}}$. The decomposition of the elastic deformation $\boldsymbol{F}_{\mathrm{E}}$ into the right elastic stretch tensor $\boldsymbol{U}_{\mathrm{E}}$ and an orthogonal part $\boldsymbol{R}_{\mathrm{E}}$ is in analogy to the decomposition of the total deformation gradient $\boldsymbol{F}=\boldsymbol{R} \boldsymbol{U}$ into a symmetric stretch tensor $\boldsymbol{U}$ and an orthogonal tensor $\boldsymbol{R}$. For the YSDH model, any texture-related effects are neglected here, in which case the plastic spin $\boldsymbol{W}_{\mathrm{P}}:=\operatorname{skw}\left(\boldsymbol{L}_{\mathrm{P}}\right)$, with $\boldsymbol{L}_{P}$, the inelastic velocity gradient given by $\dot{\boldsymbol{F}}_{\mathrm{P}} \boldsymbol{F}_{\mathrm{P}}^{-1}$, is neglected. Consequently, the evolution of $\boldsymbol{R}_{\mathrm{E}}$ is given by the Jaumann form

$\dot{\boldsymbol{R}}_{\mathrm{E}}=\boldsymbol{W} \boldsymbol{R}_{\mathrm{E}}$.

Here, $\boldsymbol{W}:=\operatorname{skw}(\boldsymbol{L})$ represents the continuum spin, i.e., the skew-symmetric part of the velocity gradient $\boldsymbol{L}=\dot{\boldsymbol{F}} \boldsymbol{F}^{-1}$. In what follows, we will also work with the rate of deformation $\boldsymbol{D}:=\operatorname{sym}(\boldsymbol{L})$.

As is standard in the context of the multiplicative decomposition of $\boldsymbol{F}$, constitutive equations are formulated in the intermediate configuration, i.e., relative to $\boldsymbol{F}_{\mathrm{P}}$. Neglecting any texture effects, as well as any inelastic volume changes due to processes such as damage, phase transformations, etc., the elastic behavior of the model is determined by the isotropic forms

$$
\begin{aligned}
\overline{\operatorname{tr}(\boldsymbol{M})} & =3 \kappa \operatorname{tr}(\boldsymbol{D}), \\
\overline{\operatorname{dev}(\boldsymbol{M})} & =2 \mu\left\{\boldsymbol{R}_{\mathrm{E}}^{\mathrm{T}} \operatorname{dev}(\boldsymbol{D}) \boldsymbol{R}_{\mathrm{E}}-\boldsymbol{D}_{\mathrm{P}}\right\},
\end{aligned}
$$

for the evolution of the trace $\operatorname{tr}(\boldsymbol{M})$ and deviatoric part $\operatorname{dev}(\boldsymbol{M})$, respectively, of the Mandel stress $\boldsymbol{M}[9,16]$. Here, $\kappa$ and $\mu$ represent the elastic bulk and shear modulus, respectively. In the context of small elastic strain, the Mandel stress determines the Kirchhoff stress $\boldsymbol{K}$ via

$\boldsymbol{K} \approx \boldsymbol{R}_{\mathrm{E}} \boldsymbol{M} \boldsymbol{R}_{\mathrm{E}}^{\mathrm{T}}$.

In particular, this implies

$\operatorname{tr}(\boldsymbol{K})=\operatorname{tr}(\boldsymbol{M}), \quad \operatorname{dev}(\boldsymbol{K})=\boldsymbol{R}_{\mathrm{E}} \operatorname{dev}(\boldsymbol{M}) \boldsymbol{R}_{\mathrm{E}}^{\mathrm{T}}$.

Consequently, only the deviatoric part of $\boldsymbol{K}$ is determined constitutively by $\boldsymbol{R}_{\mathrm{E}}$.

In this framework, then, the material behavior of polycrystalline sheet metal during forming processes below the forming limit is predominantly determined by a changing dislocation microstructure and attendant evolving anisotropic yield behavior. As discussed in the introduction, this model is based in particular on a yield function of the form introduced by Baltov and Sawczuk [2]

$\phi=\sqrt{(\boldsymbol{M}-\boldsymbol{X}) \cdot \mathcal{A}[\boldsymbol{M}-\boldsymbol{X}]}-\sigma_{\mathrm{Y} 0}-r$

in terms of the initial yield stress $\sigma_{\mathrm{Y} 0}$. For the class of materials under consideration, the saturation (i.e., Voce) form

$\dot{r}=c_{r}\left(s_{r}-r\right) \dot{\alpha}_{\mathrm{P}}$

for the evolution of $r$ is appropriate, driven by that of the equivalent inelastic deformation $\alpha_{\mathrm{P}}$. Here, $c_{r}$ represents the rate, and $s_{r}$ the value, for saturation associated with $r$. Since $\sigma_{\mathrm{Y} 0}$ is the initial yield stress (i.e., for $\alpha_{\mathrm{P}}=0$ ), the initial value $r_{0}$ of $r$ is zero. In the current rate-independent context, $\alpha_{\mathrm{P}}$ is determined as usual by the consistency condition. Analogous to isotropic hardening, kinematic hardening is modeled via the saturation (i.e., Armstrong-Frederick) form

$\dot{\boldsymbol{X}}=c_{x}\left(s_{x} \boldsymbol{N}_{\mathrm{P}}-\boldsymbol{X}\right) \dot{\alpha}_{\mathrm{P}}$

for the evolution of $\boldsymbol{X}$ depending on corresponding (constant) saturation rate $c_{x}$, (constant) saturation magnitude $s_{x}$, as well as the (variable) direction $\boldsymbol{N}_{\mathrm{P}}:=$ $\boldsymbol{D}_{\mathrm{P}} /\left|\boldsymbol{D}_{\mathrm{P}}\right|$ of the rate of inelastic deformation

$\boldsymbol{D}_{\mathrm{P}}=\dot{\alpha}_{\mathrm{P}} \partial_{\boldsymbol{M}-\boldsymbol{X}} \phi$,

which is modeled here in associated form. The initial value of $\boldsymbol{X}$ is assumed to be zero. 
The constitutive model formulation is completed by an evolution relation for $\mathcal{A}$ in order to represent the effect of cross hardening on the material behavior. The form of this relation introduced in what follows is based on the idea that active or "dynamic" dislocation microstructures oriented with respect to the current loading direction (idealized in the model context by $\boldsymbol{N}_{\mathrm{P}}$ ) persist and become inactive or "latent" after a loadingpath change and strengthen existing obstacles to glidesystem activation in the new loading direction. In addition, both dynamic and latent dislocation structures are assumed to saturate with increasing accumulated inelastic deformation. These assumptions are built into the constitutive relation

$$
\begin{aligned}
\dot{\mathcal{A}}= & c_{d}\left(s_{d, \text { sat }} \boldsymbol{N}_{\mathrm{P}} \otimes \boldsymbol{N}_{\mathrm{P}}-\mathcal{A}_{d}\right) \dot{\alpha}_{\mathrm{P}} \\
& +c_{l}\left\{s_{l, \text { sat }}\left(\mathcal{I}_{\mathrm{dev}}-\boldsymbol{N}_{\mathrm{P}} \otimes \boldsymbol{N}_{\mathrm{P}}\right)-\mathcal{A}_{l}\right\} \dot{\alpha}_{\mathrm{P}}
\end{aligned}
$$

for the evolution of $\mathcal{A}$. Here, $\mathcal{I}_{\mathrm{dev}}$ is the deviatoric part of the fourth-order identity tensor, and

$$
\begin{aligned}
\mathcal{A}_{d} & :=\left(\boldsymbol{N}_{\mathrm{P}} \cdot \mathcal{A} \boldsymbol{N}_{\mathrm{P}}\right) \boldsymbol{N}_{\mathrm{P}} \otimes \boldsymbol{N}_{\mathrm{P}}, \\
\mathcal{A}_{l} & :=\mathcal{A}-\mathcal{A}_{d},
\end{aligned}
$$

represent the "dynamic" and "latent" parts of $\mathcal{A}$, respectively. More precisely, these are the projections of $\mathcal{A}$ parallel and orthogonal, respectively, to the current (instantaneous) inelastic flow direction $\boldsymbol{N}_{\mathrm{P}}$. The first term in Eq. 9 is of the saturation type with respect to $\mathcal{A}_{d}$, with $c_{d}$ the rate of saturation, and $s_{d \text {, sat }} \boldsymbol{N}_{\mathrm{P}} \otimes \boldsymbol{N}_{\mathrm{P}}$ the saturation value, respectively, of $\mathcal{A}_{d}$. Likewise, $c_{l}$ is the saturation rate, and $s_{l, \text { sat }}\left(\mathcal{I}_{\mathrm{dev}}-\boldsymbol{N}_{\mathrm{P}} \otimes \boldsymbol{N}_{\mathrm{P}}\right)$ the saturation value, of $\mathcal{A}_{l}$. The initial value $\mathcal{A}_{0}$ of $\mathcal{A}$ is determined by any Hill initial flow orthotropy due to any texture from rolling.

The current material model was implemented in the commercial FE codes Abaqus and LS-Dyna via the user material interfaces provided. Besides the two elasticity parameters $\kappa, \mu$ and the six parameters (e.g., in the sense of Hill: $F, G, H, L, M, N$ ) for the initial flow orthotropy, this model contains eight hardening parameters $c_{r}, s_{r}, c_{x}, s_{x}, c_{d}, s_{d \text {, sat }}, c_{l}, s_{l, \text { sat }}$ to be identified using the tests described in the mechanical test section.

\section{Single crystal plasticity model}

As a second approach, a microscopic model is formulated in the context of elasto-viscoplastic single crystal plasticity. Since the focus here lies on the observation of dislocation structures, in this work the model behavior at the single grain level is emphasized. A fullconstraints Taylor model is implemented in order to represent the behavior of the polycrystal. However, the model presentation is restricted to the single crystal level.

As in the previous model the multiplicative decomposition of $\boldsymbol{F}$ into elastic and plastic parts, $\boldsymbol{F}_{\mathrm{E}}$ and $\boldsymbol{F}_{\mathrm{P}}$, respectively, is used. In contrast to the engineering scale model the plastic spin is non-zero. As usual in the context of crystal plasticity, the plastic part of the velocity gradient is given by

$\boldsymbol{L}_{\mathrm{P}}=\sum_{a} \dot{\gamma}_{a} \boldsymbol{s}_{a} \otimes \boldsymbol{n}_{a}=\sum_{a} \operatorname{dir}\left(\tau_{a}\right) \boldsymbol{s}_{a} \otimes \boldsymbol{n}_{a} \dot{\alpha}_{a}$.

$\boldsymbol{s}_{a}$ and $\boldsymbol{n}_{a}$ represent the glide direction and glide plane normal of the $a$-th glide system, respectively. $\tau_{a}$, the Schmid stress is given by

$\tau_{a}:=\boldsymbol{s}_{a} \cdot \boldsymbol{M n}_{a}$.

Differing from other crystal plasticity formulations, $\gamma_{a}$ is interpreted as the glide system shear, which can be positive or negative and decrease or increase. Here, the constitutive assumption $\operatorname{dir}\left(\dot{\gamma}_{a}\right)=\operatorname{dir}\left(\tau_{a}\right)$ is used for the direction of the glide-system shear-rate and no differentiation between $\boldsymbol{s}_{a},-\boldsymbol{s}_{a}$ as glide directions is used. In the fcc case with $\{111\}\langle 110\rangle$ glide systems this leads to 12 distinct glide systems. The glide system flow rule is given by

$\dot{\gamma}_{a}=\dot{\gamma}_{0} \operatorname{sign}\left(\tau_{a}\right)\left|\frac{\tau_{a}}{\tau_{c a}}\right|^{m_{0}}$,

with $\tau_{c a}$ the critical shear stress on glide system $a$ and $\dot{\gamma}_{0}$ a referential slip rate and $m_{0}$ the visco-plastic exponent. Here, the constituitive assumption that the direction of gliding is determined by the direction of the applied shear stress $\operatorname{sign}\left(\tau_{a}\right)$. In analogy to the YSDH model the accumulated inelastic glide system shear rate is defined as $\dot{\alpha}_{a} \equiv\left|\dot{\gamma}_{a}\right|$. Similar to the previous model, incremental updates for the elastic rotation $\boldsymbol{R}_{\mathrm{E}}$, the elastic GreenLagrange strain $\boldsymbol{E}_{\mathrm{E}}$, the plastic strain tensor $\boldsymbol{\Delta}_{\mathrm{P}}$ and its counterpart the plastic spin vector $\omega_{\mathrm{P}}$ according to the framework outlined in $[17,18]$ are performed in the local predictor-corrector scheme.

The single crystal plasticity hardening rule is adopted according to the one proposed by Holmedal et al. [12]. There are three distinct contributions to hardening on the glide system level influencing the critical shear stress $\tau_{c a}$ in the Holmedal model

$\tau_{c a}=\tau_{r}+\tau_{d a}+\tau_{l a}$

The isotropic hardening term $\tau_{r}$ is modeled by a phenomenological work-hardening rule and influences all glide systems equally. Note that in the original model this term was referred to as $\tau_{\mathrm{i}}$. In order to emphasize the analogy with the YSDH model all terms related 


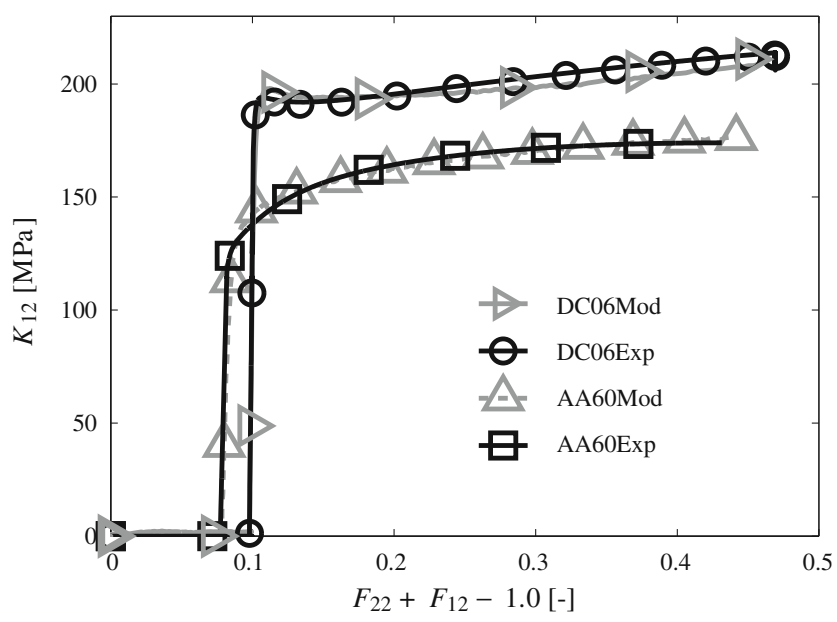

Fig. 11 Plane tension to shear experiment vs YSDH model for the experiments shown in Figs. 2 and 3. DC06Mod and DC06Exp represent the stress-strain curves for the YSDH model and the DC06 experiment(s), respectively. Likewise AA60Mod and AA60Exp stand for the AA6016-T4 case

to isotropic hardening are denoted by index $r . \tau_{l a}$ accounts in a phenomenological fashion for the hardening related to persistent dislocation structures which will become apparent if changes in active glide systems occur. In the model the corresponding hardening term $\tau_{l a}$ affects only latent glide systems similar as $\mathcal{A}_{l}$ in the YSDH model. The term $\tau_{d a}$ represents an extra contribution of hardening associated with active glide systems. Holmedal et al. used the form for the isotropic hardening contribution

$\tau_{r}=\tau_{0}+s_{r, \mathrm{II}}\left(1-e^{-c_{r, \mathrm{II}} \alpha}\right)+s_{r, \mathrm{III}}\left(1-e^{-c_{r, \mathrm{III}} \alpha}\right)+r_{\mathrm{IV}} \alpha$.

Here, $\tau_{0}, s_{r, \text { II }}, c_{r, \text { II }}, s_{r, \text { III }}, c_{r \text {,III }}$ and $r_{\text {IV }}$ are material parameters to be determined from experimental data and related to stage II, III and IV hardening. The hardening rules for the dynamic and latent part are given by

$\dot{\tau}_{d a}=c_{d}\left(\tau_{d a}^{\mathrm{sat}}-\tau_{d a}\right) \dot{\alpha}$ and

$\dot{\tau}_{l a}=c_{l}\left(\tau_{l a}^{\mathrm{sat}}-\tau_{l a}\right) \dot{\alpha}$,

respectively. $c_{d}$ and $c_{l}$ are material parameters governing the evolution rates of the directional and latent parts, similar to those in the YSDH model. The satu- ration values $\tau_{d a}^{\text {sat }}$ and $\tau_{l a}^{\text {sat }}$ are dependent on the activity of the corresponding glide system and given by

$\tau_{d a}^{\mathrm{sat}}=\left\{\begin{array}{ll}q_{d} \tau_{r} & \dot{\alpha}_{a} \geq \dot{\alpha}_{\mathrm{c}} \\ 0 & \dot{\alpha}_{a}<\dot{\alpha}_{\mathrm{c}}\end{array}\right.$,

such that the saturation value $\tau_{d a}^{\text {sat }}$ evolves only for active glide systems.

$\tau_{l a}^{\mathrm{sat}}=\left\{\begin{array}{ll}q_{l} & \tau_{r} \\ \dot{\alpha}_{a} \leq \dot{\alpha}_{\mathrm{c}} \\ 0 & \dot{\alpha}_{a}>\dot{\alpha}_{\mathrm{c}}\end{array}\right.$.

Contrary the saturation value $\tau_{l a}^{\text {sat }}$ evolves only for nonactive systems.

In Eqs. 17 and 18, $q_{d}$ and $q_{l}$ are additional material parameters governing the rate of evolution of $\tau_{d a}^{\text {sat }}$ and $\tau_{l a}^{\text {sat }}$, respectively. $\dot{\alpha}_{\mathrm{c}}$ is a threshold value which had to be introduced due to the fact that unlike in the original Holmedal model no work minimization of the rate of plastic work is performed in order to determine the active glide systems. In addition it has to be noted, that in the current implementation, a hardening term $\tau_{\mathrm{r} a}$ included in the original model accounting for changes in the critical shear stress namely softening upon reversal of glide systems is neglected here, since the focus lies on cross-hardening.

\section{Parameter identification}

The parameter identification for the YSDH model based on the stress-strain data obtained from uniaxial and plane strain tension, monotonic shear, forward shear to reverse shear and finally plane strain tension to simple shear tests is described briefly. Parameters for the Holmedal model are adopted from [12,14].

Macroscale metal plasticity model

Since isotropic, kinematic and directional hardening are decoupled in the YSDH model, this feature can be exploited in the identification procedure for the whole set of parameters by determining subsets succesively. In the procedure described in detail in [26], in the first step $s_{r}$ and $c_{r}$ governing the isotropic hardening are determined based on the monotonic tests. Then the two parameters $s_{x}$ and $c_{x}$ responsible for kinematic

Table 3 Identified YSDH model parameter values for DC06 and AA6016-T4; parameter values determined from uniaxial tension, monotonic shear, cyclic shear, and orthogonal tension-shear, test data

\begin{tabular}{llllllll}
\hline Item & $s_{r}$ & $c_{r}$ & $s_{x}$ & $c_{x}$ & $s_{d, \text { sat }}$ & $c_{d}$ & $s_{l, \text { sat }}$ \\
\hline DC06 & $192 \mathrm{MPa}$ & 6.64 & $56.0 \mathrm{MPa}$ & 33.1 & 0.0 & 23.9 & -0.447 \\
AA6016-T4 & $118 \mathrm{MPa}$ & 8.96 & $37.8 \mathrm{MPa}$ & 56.0 & 0.0 & 13.9 & -0.0910 \\
\hline
\end{tabular}


Table 4 Hardening parameters for the Holmedal model for AA3103[12]

\begin{tabular}{|c|c|c|c|c|c|c|c|c|c|c|}
\hline Item & $\tau_{0}$ & $s_{r, \mathrm{II}}$ & $c_{r, \mathrm{II}}$ & $s_{r, \mathrm{III}}$ & $c_{r, \mathrm{III}}$ & $r_{\mathrm{IV}}$ & $q_{l}$ & $q_{d}$ & $c_{l}$ & $c_{d}$ \\
\hline AA3103 & $10 \mathrm{MPa}$ & $10.45 \mathrm{MPa}$ & 18.2 & $14 \mathrm{MPa}$ & 1 & $2.5 \mathrm{MPa}$ & 0.6 & 0.35 & 4 & 100 \\
\hline
\end{tabular}

hardening are determined based on the reverse tests. Finally, the four parameters $s_{d \text {, sat }}, c_{d}, s_{l \text {, sat }}$ and $c_{l}$ representing the influence of distorsional hardening are determined based on the cross-test. Here, it has to be noted that $s_{d \text {, sat }}$ actually can be determined from an a priori estimate by exploiting the characteristics of the model and the observed hardening behavior of the material as shown in detail in [26]. The material parameter determination is carried out using the program LS-OPT in conjunction with LS-DYNA. Given the homogeneous nature of the tests, one-element calculations suffice. The optimization technique used relies on response surface methodology [31]. For DC06 the identification is based on the fixed values $\kappa=151 \mathrm{GPa}$ and $\mu=69.6 \mathrm{GPa}$ for the elastic properties, as well as that $\sigma_{\mathrm{Y} 0}=132 \mathrm{MPa}$ for the initial yield stress, all at room temperature. The Hill parameters $F=0.259$, $G=0.302, H=.698$ and $N=1.36$ are determined on the basis of the average $r_{0}, r_{45}$ and $r_{90}$ values mentioned in the materials section. Strictly speaking, only $N, F$, $G$ and $H$ can be determined by in-plane tensile tests. For through thickness shear, isotropy is tacitly assumed, resulting in $L=M=1.5$. The corresponding values for AA6016-T4 are $\kappa=66.7 \mathrm{GPa}$ and $\mu=25.6 \mathrm{GPa}$, $\sigma_{\mathrm{Y} 0}=105 \mathrm{MPa}, F=0.502, G=0.614, H=0.387$ and $N=1.02$.

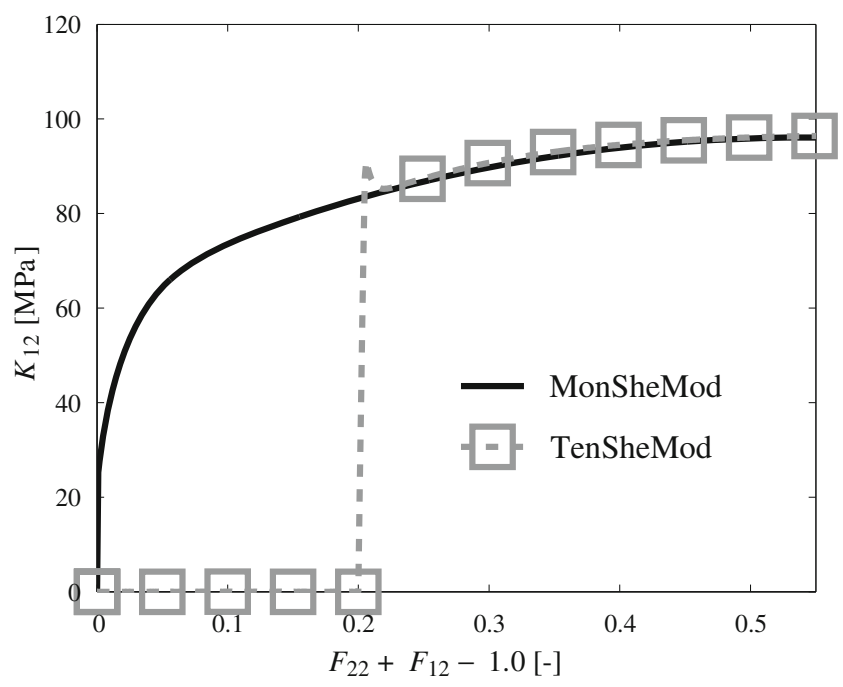

Fig. 12 Monotonic simple shear test and uniaxial tension to simple shear test with a pre-strain of $20 \%$ for a single fcc crystal with the [100] direction oriented in the tension direction and shear direction oriented in [010] for the single crystal plasticity model using the parameters given in Table 4
As can be seen from Fig. 11 by comparison of the experimental curves with the stress-strain curves predicted by the YSDH model the general agreement for both materials is good. The parameter values for $s_{x}$ for the saturation value of 56.0 and $37.8 \mathrm{MPa}$ for the backstress for DC06 and AA6016-T4, respectively, indicate that the contribution of kinematic hardening to the overall hardening behavior is less distinct in AA6016-T4 since $s_{x}$ is smaller for AA6016-T4 than for DC06 (Table 3). This coincides with the observations briefly mentioned in the mechanical test section. A similar statement can be made for the influence of distortional hardening. The magnitude of the saturation value of $s_{l, \text { sat }}$ is about factor 5 smaller for AA6016-T4 than for DC06. This corresponds to the smaller amount of cross-hardening observed in the mechanical tests and to the less pronounced persistent dislocation structure observed in the sections discussing the mechanical testing and the microstructural investigations.

\section{Single crystal plasticity model}

The elastic constants $C_{11}=107 \mathrm{GPa}, C_{12}=54.7 \mathrm{GPa}$ and $C_{44}=26 \mathrm{GPa}$ for aluminum are adopted from [1]. For the referential shear rate $\dot{\gamma}_{0}=0.001 \mathrm{~s}^{-1}$ is used, together with a viscoplastic exponent $m_{0}=2.25$. The values for the hardening behavior of AA3103 are adopted from [12] and given in Table 4. Combinations of plane strain compression to uniaxial compression tests in different directions with respect to the rolling direction, transverse and normal direction were used to investigate the material behavior of AA3103. Holmedal et al. [12] used stacks of glued sheets in combination with a channel die to obtain these parameters in monotonic, quasi-reverse and quasi-cross tests.

Figure 12 shows that a small increase in shear stress can be observed upon the change of the deformation mode based on the model prediction. As is the case for AA6016-T4 the effect is less distinct than in DC06.

\section{Model comparison and discussion}

As the model descriptions show, the two models share similarities in the approach how cross-hardening is represented from a mathematical point of view. The fact that both models use terms, separating contributions from latent slip systems or in the YSDH 
model, non-active directions of inelastic flow, from those of currently active slip systems/flow directions, is owed to the findings at the microstructural scale touched in the microstructural investigation section. The exact mechanisms of the formation of cells and cell-block-boundaries and the corresponding transient stress-strain behavior are, at least, not fully understood. Thus, phenomenological modeling of the microstructural evolution at different scales offers the means to capture the main characteristics of material behavior. From the point of modeling, it it interesting to see how this is achieved. Taking a look at Figs. 13 and 14 showing the evolution of internal variables for both models for the case of a tension-shear experiment helps to do so.

Beginning with the YSDH model one can see that $a_{d}$ which is associated with the current direction of inelastic flow in the model and thus with the currently active set of glide systems on the microscopic scale, hardly evolves for any monotonic deformation, here represented by the first tension stage. Instead, $\left|\mathcal{A}_{l}\right|$, which is associated with directions orthogonal to the current direction of inelastic flow, evolves towards its saturation value during monotonic deformation. In the YSDH model this can be interpreted as the strength of latent dislocation structures and the corresponding latent hardening which is present irrespective of the occurrence of a strain-path change. In mechanical tests the effect of the latent hardening, the immediate increase of the yield stress, only becomes apparent after an orthogonal or quasi-orthogonal change of strainpath which is reflected in the YSDH model by the fact

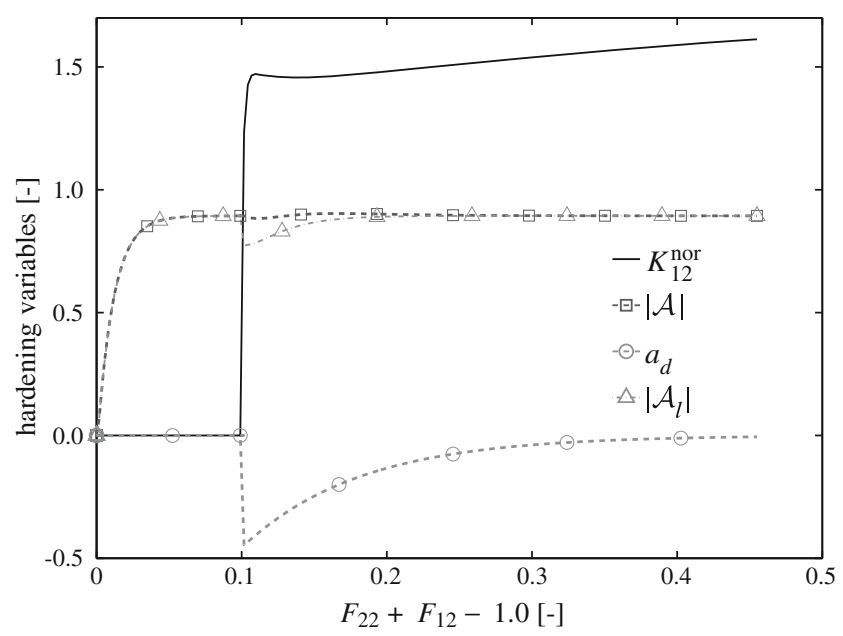

Fig. 13 Evolution of internal variables for tension-shear experiment for DC06 over $F_{22}+F_{12}-1.0$ predicted by YSDH. For comparison purposes the normalized shear stress $K_{12}^{\text {nor }}=$ $K_{12} / \sigma_{\mathrm{Y} 0}$ is also shown

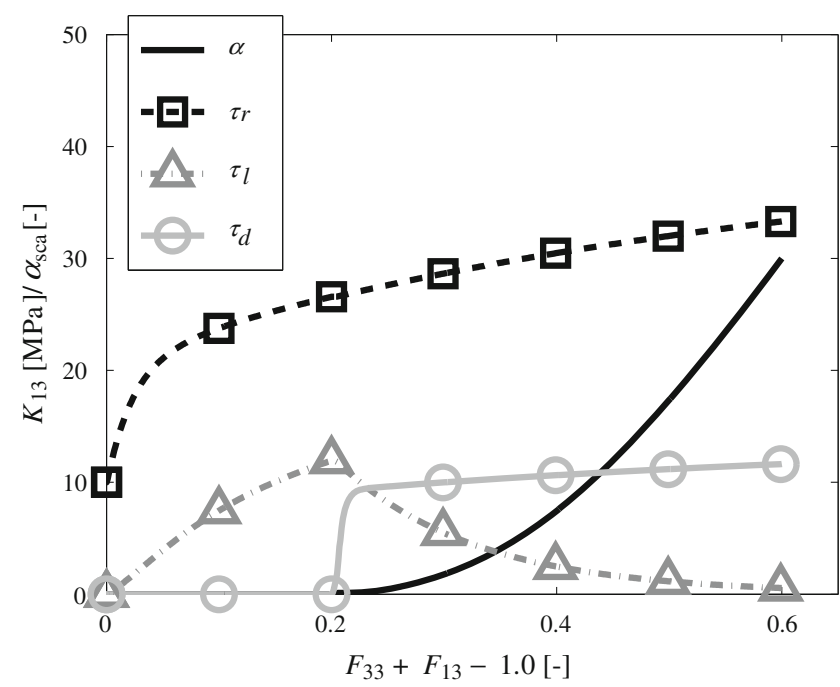

Fig. 14 Evolution of internal variables $\tau_{r}, \tau_{l}, \tau_{d}$ for an uniaxial tension to simple shear test with a pre-strain of $20 \%$ for a single fcc crystal with the [100] direction aligned with the global 1direction and tension applied in the [001] direction. The evolution of the variables is shown for the (-1-11) [1 - 10] glide system. Shear direction is [100]. The material parameters are given in Table 4. $\alpha$ is the corresponding accumulated glide system shear shown for comparison

that the yield surface is distorted in directions orthogonal to the currently active stress, shown e.g. in [26]. The stress state and thus the deformation state decide whether this has a distinct effect on the resulting stressstrain behavior. This is very similar to the Holmedal model where now the crystallographic slips are used as model quantities. Figure 14 shows the hardening contributions $\tau_{r}, \tau_{l}$ and $\tau_{d}$ for a virtual tension-shear experiment performed on a single crystal whose crystallographic axes are aligned with the global axes. The evolution of the variables is shown for a single representative glide system changing from inactive to active upon the change in deformation mode as indicated by the scaled accumulated slip $\alpha$. As in the YSDH model the directional term $\tau_{d}$ is zero, as long as the glide system is non-active and tends towards its saturation value. Contrary, the latent contribution $\tau_{l}$ increases for the non-active phase as in the YSDH and then tends towards zero, representing the vanishing influence of the now annihilated dense-dislocation walls.

Besides those similarities, there are of course differences in the two models, the major being related to the chosen scale and such to the computational effort. But both types of models may serve different purposes. The Holmedal model is clearly aimed at being used for fcc materials where texture evolutions and thus the texture simulation play a more important role than in bcc materials. However such a model, once 
being calibrated may be used to investigate the yield surface evolution for experimentally-hard to obtain stress states. This information may be used to enhance models such as the YSDH which are closer related to larger scale simulations of technical processes.

\section{Outlook}

Further TEM-investigations and corresponding electron-back-scatter-diffraction measurements of the evolution of the texture at least for the fcc case are expected to yield a better understanding of the interaction of grain and dislocation microstructure. As stated earlier, the influence of the texture evolution is expected to have a larger influence on the mechanical response of AA6016-T4 than for the interstitial free steel. Also, in-situ experiments of micro-specimen giving full insight into the formation history of such dislocation structures seen in this work seem desirable to intensify the development of models on this scale.

Acknowledgements Financial support for this work provided by the German Science Foundation (DFG) under contract PAK 250 is greatly acknowledged. The DC06 material investigated for this paper was provided and chemically analyzed by ThyssenKrupp Steel Europe AG. The AA6016-T4 material was provided by Novelis. The authors like to thank Maarten van Riel (Faculty of Engineering Technology, University of Twente, the Netherlands) for his guidance with the biaxial tester.

Open Access This article is distributed under the terms of the Creative Commons Attribution Noncommercial License which permits any noncommercial use, distribution, and reproduction in any medium, provided the original author(s) and source are credited.

\section{References}

1. Balasubramanian S, Anand L (2002) Elasto-viscoplastic constitutive equations for polycrystalline fcc materials at low homologous temperatures. J Mech Phys Solids 50(1):101-126

2. Baltov A, Sawczuk A (1965) A rule of anisotropic hardening. Acta Mech I(2):81-92

3. Bouvier S, Teodosiu C, Haddadi H, Tabacaru V (2003) Anisotropic work-hardening behaviour of structural steels and aluminium alloys at large strains. J Phys IV France 105:215-222

4. Bouvier S, Alves J, Oliveira M, Menezes L (2005) Modelling of anisotropic work-hardening behaviour of metallic materials subjected to strain-path changes. Comput Mater Sci 32:301-315

5. de Montleau P (2004) Programming of Teodosiu's hardening model. IAP P5/08 progress report, Mechanical Engineering, University of Liege, Belgium

6. Estrin Y, Tóth LS, Molinari A, Bréchet Y (1998) A dislocation-based model for all hardening stages in large strain deformation. Acta Mater 46(15):5509-5522
7. Fernandes JV, Schmitt JH (1983) Dislocation microstructures in steel during deep drawing. Philos Mag A 48(6): $841-870$

8. Ghosh AK, Backofen WA (1973) Strain-hardening and instability in biaxially stretched sheets. Metall Trans 4:11131123

9. Gurtin M, Fried E, Anand, L (2010) The mechanics and thermodynamics of continua. Cambridge University Press, Cambridge

10. Hasegawa T, Yakou T, Karashima S (1975) Deformation behaviour and dislocation structures upon stress reversal in polycrystalline aluminium. Mater Sci Eng 20:267-276

11. Hiwatashi S, van Bael A, van Houtte P, Teodosiu C (1997) Modelling of plastic anisotropy based on texture and dislocation structure. Comput Mater Sci 9:274-284

12. Holmedal B, van Houtte P, An Y (2008) A crystal plasticity model for strain-path changes in metals. Int J Plast 24(8):1360-1379

13. Hu Z, Rauch EF, Teodosiu C (1992) Work-hardening behavior of mild steel under stress reversal at large strains. Int J Plast 8(7):839-856

14. Kalidindi SR, Bronkhorst CA, Anand L (1992) Crystallographic texture evolution in bulk deformation processing of fcc metals. J Mech Phys Solids 40(3):537-569

15. Li S, Hoferlin E, van Bael A, van Houtte $\mathrm{P}$, Teodosiu C (2003) Finite element modeling of plastic anisotropy induced by texture and strain-path change. Int J Plast 19:647674

16. Mandel J, Généralization de la théorie de plasticité de W. T. Koiter. Int J Solid Struct 1:273-29

17. Marin EB, Dawson PR (1998) Elastoplastic finite element analyses of metal deformations using polycrystal constitutive models. Comput Methods Appl Mech Eng 165(1-4): 23-41

18. Marin EB, Dawson PR (1998) On modelling the elastoviscoplastic response of metals using polycrystal plasticity. Comput Methods Appl Mech Eng 165(1-4):1-21

19. Mughrabi H (1983) Dislocation wall and cell structures and long-range internal stresses in deformed metal crystals. Acta Metall 31(9):1367-1379

20. Deutsches Institut für Normung e.V. (2006) DIN EN 10049measurement of roughness average $\mathrm{Ra}$ and peak count RPc on metallic flat products. Tech. rep., DIN

21. Deutsches Institut für Normung e.V. (2006) DIN EN 10130cold rolled low carbon steel flat products for cold forming technical delivery conditions. Tech. rep., DIN

22. Deutsches Institut für Normung e.V. (2009) DIN EN 573-3 aluminium and aluminium alloys - chemical composition and form of wrought products. Tech. rep., DIN

23. Deutsches Institut für Normung e.V. (2009) DIN EN ISO 6892-1 metallic materials-tensile testing-part 1: method of test at room temperature. Tech. rep., DIN

24. Nesterova EV, Bacroix B, Teodosiu C (2001) Experimental observation of microstructure evolution under strain-path changes in low-carbon if steel. Mater Sci Eng A 309-310:495499

25. Nesterova EV, Bacroix B, Teodosiu C (2001) Microstructure and texture evolution under strain-path changes in lowcarbon interstitial-free steel. Metall Mater Trans 32A:25272538

26. Noman M, Clausmeyer T, Barthel C, Svendsen B, Huétink J, van Riel M (2010) Experimental characterization and modeling of the hardening behavior of the sheet steel LH800. Mater Sci Eng A 527(10-11):2515-2526

27. Peeters B, Kalidindi SR, Teodosiu C, van Houtte P, Aernoudt E (2002) A theoretical investigation of the 
influence of dislocation sheets on evolution of yield surfaces in single-phase b.c.c. polycrystals. J Mech Phys Solids 50(4):783-807

28. Rauch EF, Schmitt JH (1989) Dislocation substructures in mild steel deformed in simple shear. Mater Sci Eng A 113:441-448

29. Rauch EF, Thuillier S (1993) Rheological behaviour of mild steel under monotonic loading conditions and cross-loading. Mater Sci Eng A 164:255-259

30. Rybin VV (1986) Severe plastic deformations and fracture of metals. Metallurgiya, Moscow

31. Stander N, Roux W, Goel T, Eggleston T, Craig K (2008) LS-OPT user's manual. Livermore Software Technology Corporation

32. Strauven Y, Aernoudt E (1987) Directional strain softening in ferritic steel. Acta Metall 35:1029-1036

33. Teodosiu C, Hu Z (1995) Evolution of the intragranular microstructure at moderate and large strains: modelling and computational significance. In: Shen SF, Dawson PR (eds) Simulation of materials processing: theory, methods and applications. Balkema, Rotterdam, pp 173182

34. Teodosiu C, Hu Z (1998) Microstructure in the continuum modelling of plastic anisotropy. In: Proceedings of 19th Ris $\varnothing$ international symposium on material's science: modelling of structure and mechanics of materials from microscale to product. Ris $\varnothing$ National Laboratory, Roskilde, Denmark, pp 149-168

35. Thuillier S, Rauch EF (1994) Development of microbands in mild steel during cross loading. Acta Metall Mater 42:19731983

36. Thuillier S, Manach PY, Menezes LF (2010) Occurence of strain path changes in a two-stage deep drawing process. J Mater Process Technol 210(2):226-232

37. van Riel M, van den Boogaard AH (2007) Stress-strain responses for continuous orthogonal strain path changes with increasing sharpness. Scr Mater 57(5):381-384

38. van Riel M (2009) Strain path dependency in sheet metal-experiments and models. Dissertation, Universiteit Twente 